\title{
Umbilical Cord-Derived Mesenchymal Stem Cells for Hematopoietic Stem Cell Transplantation
}

\author{
Yu-Hua Chao, ${ }^{1,2}$ Han-Ping Wu, ${ }^{3}$ Chin-Kan Chan, ${ }^{4}$ Chris Tsai, ${ }^{5}$ \\ Ching-Tien Peng, ${ }^{6,7,8}$ and Kang-Hsi Wu ${ }^{7,8}$ \\ ${ }^{1}$ Department of Pediatrics, Chung Shan Medical University Hospital, Taichung, Taiwan \\ ${ }^{2}$ School of Medicine, Chung Shan Medical University, Taichung, Taiwan \\ ${ }^{3}$ Department of Pediatrics, Buddhist Tzu-Chi General Hospital, Taichung Branch, Taichung, Taiwan \\ ${ }^{4}$ Department of Pediatrics, Taoyuan General Hospital, Taoyuan, Taiwan \\ ${ }^{5}$ Bionet Corporation, Taipei, Taiwan \\ ${ }^{6}$ Department of Biotechnology and Bioinformatics, Asia University, Taichung, Taiwan \\ ${ }^{7}$ School of Chinese Medicine, China Medical University, Taichung, Taiwan \\ ${ }^{8}$ Department of Pediatrics, China Medical University Hospital, Taichung, Taiwan
}

Correspondence should be addressed to Kang-Hsi Wu, d5284@mail.cmuh.org.tw

Received 18 July 2012; Accepted 31 July 2012

Academic Editor: Somayeh Shahrokhi

Copyright () 2012 Yu-Hua Chao et al. This is an open access article distributed under the Creative Commons Attribution License, which permits unrestricted use, distribution, and reproduction in any medium, provided the original work is properly cited.

Hematopoietic stem cell transplantation (HSCT) is becoming an effective therapeutic modality for a variety of diseases. Mesenchymal stem cells (MSCs) can be used to enhance hematopoietic engraftment, accelerate lymphocyte recovery, reduce the risk of graft failure, prevent and treat graft-versus-host disease, and repair tissue damage in patients receiving HSCT. Till now, most MSCs for human clinical application have been derived from bone marrow. However, acquiring bone-marrow-derived MSCs involves an invasive procedure. Umbilical cord is rich with MSCs. Compared to bone-marrow-derived MSCs, umbilical cord-derived MSCs (UCMSCs) are easier to obtain without harm to the donor and can proliferate faster. No severe adverse effects were noted in our previous clinical application of UCMSCs in HSCT. Accordingly, application of UCMSCs in humans appears to be feasible and safe. Further studies are warranted.

\section{Introduction}

Biologic interest in mesenchymal stem cells (MSCs), which were first described by Friedenstein and colleagues in 1966 [1], has risen dramatically over the last decade. As expected, clinical application of human MSCs is also evolving rapidly for a variety of diseases. Bone marrow (BM) remains a major source of MSCs in most investigations. Although umbilical cord (UC) rich with MSCs and UC-derived MSCs (UCMSCs) has been shown to be easy to isolate and culture, data available focusing on clinical application of UCMSCs are quite limited.

In this paper, we address the characteristics of MSCs, including UCMSCs, and their clinical application in hematopoietic stem cell transplantation (HSCT). We share our clinical experience in the successful use of in vitro expanded
UCMSCs [2-4]. We also describe several issues related to the clinical use of MSCs. For the near future, we anticipate that more patients can benefit from the therapeutic effects of UCMSCs.

\section{Biologic Characteristics of MSCs}

2.1. The Definition of MSCs. The defining characteristics of MSCs are inconsistent among investigators. According to the minimal criteria of the International Society for Cellular Therapy, MSCs are defined by their in vitro growth pattern, the expression of specific surface antigens, and the multipotent differentiation potential [5]. Being a member of stem cells, MSCs are able to self-renew with a high proliferative capacity. They proliferate as fibroblastic spindle-shaped cells and must be plastic-adherent when maintained in standard 
culture conditions [5]. MSCs can be characterized by a panel of surface markers, which is negative for hematopoietic antigens (CD34, CD45, CD14, CD11b, CD19, and CD79 $\alpha$ ), and positive for mesenchymal makers (CD105 and CD73) and cell adhesion molecules (CD29, CD44, CD106, and CD90) [5-7]. MSCs display a broader differentiation potential, and the most unique property to identify MSCs is the capacity for trilineage mesenchymal differentiation, including osteoblasts, adipocytes, and chondroblasts [5].

2.2. The Role of MSCs in Hematopoiesis. MSCs play a critical role in providing the essential microenvironment for hematopoiesis. In BM, MSC-derived stromal cells establish an appropriate scaffold and a complex network of cytokines, adhesion molecules, and extracellular matrix proteins $[8,9]$. In vitro, many studies have reported the promotive effects of MSCs for expansion of hematopoietic stem cells (HSCs) [1012]. In animal models, MSCs have been shown to enhance engraftment of donor HSCs after cotransplantation [13-15]. Like BM-derived MSCs (BMMSCs), UCMSCs were demonstrated to support engraftment of a limited number of human HSCs from cord blood in a NOD/SCID transplant model [16].

2.3. Immune Properties of MSCs. MSCs express HLA-class I, but not class II $[17,18]$. In vitro, several investigations have demonstrated that using mismatched MSCs does not trigger proliferative T-cell response in the allogeneic mixed lymphocyte reaction $[12,18,19]$. In vivo, transplanted allogeneic MSCs can be detected in recipients at extended time points, indicating a lack of immune recognition and clearance [20]. As for showing low immunogenic properties, it is convenient for the clinical application of MSCs.

MSCs possess immunomodulatory effects. Many studies have reported that MSCs can exert profound immunosuppressive effects via modulation of both cellular and innate immune pathways. MSCs suppress the proliferative response of $B$ cells to allogenic antigens through an arrest in the $G_{0} / G_{1}$ phase of the cell cycle, and the major mechanism appears to be through soluble factors produced by MSCs [21-23]. MSCs also inhibit B-cell differentiation and affect their chemotactic properties [23]. Additionally, MSCs modify Tcell responses indirectly by blocking the differentiation of monocytes into dendritic cells and impairing the antigenpresenting ability [24]. MSCs also inhibit T-cell proliferation and function directly by secreting soluble factors [25-27]. Moreover, MSCs alter the cytokine secretion profiles of dendritic cells, $\mathrm{T}$ cells, and natural killer cells to induce a more anti-inflammatory or tolerant phenotype [20].

Taken together, MSCs are capable of escaping recognition by the alloreactive immune system and can exert immunomodulatory and anti-inflammatory effects. These properties make them a promising tool in the management of graft failure and in the prevention or treatment of graft-versushost disease (GVHD) after HSCT.

\section{Isolation of MSCs from Various Origins}

MSCs are derived from mesodermal progenitor cells. They can be isolated from different tissues, including BM, peripheral blood, adipose tissue, dental pulp, and a variety of fetal tissues, such as amniotic fluid, amniotic membrane, placenta, cord blood, and UC [7, 8, 17, 28-30]. A great impact on clinical utility is related to the abundance and isolation efficacy of MSCs from different sources. Though as a traditional source, MSCs constitute only a small percentage about one in $3.4 \times 10^{4}$ cells in adult BM [31]. The frequency of MSCs in fetal tissues is likely to be higher [30]. However, MSCs are sparse in full-term infants' cord blood [31-33], whereas MSCs can be obtained and cultured much more efficiently from UC than from cord blood [34].

The ontological and anatomical origins of MSCs have profound influences on their properties, and hence affect their performance in clinical application. Many studies have found that MSCs derived from various origins share a similar spindle-shaped morphology and reveal a consistent immunophenotypic profile $[6,17,30,35]$. Whereas compared with adult-type MSCs, fetal-type MSCs appear to have greater expansion capacity and faster doubling time which may result from their longer telomeres, greater telomerase activity, and higher expression of telomerase reverse transcriptase $[6,17,28,29,36-38]$. UCMSCs shares similar properties unique to fetal-type MSCs, and we and other authors also demonstrated the higher proliferative potential of UCMSCs [3, 39, 40].

Fetal-type MSCs are less lineage-committed [36] and express lower levels of HLA-class I than adult-type MSCs [6]. We found that the reduction in peripheral blood mononuclear cell proliferation activated by phytohemagglutinin stimulation was significantly greater in the UCMSC cocultures than in BMMSC cocultures, suggesting the greater immunosuppressive effects of UCMSCs [3]. Additionally, MSCs from various origins have heterogeneity in differentiation potential. For example, MSCs from UC or cord blood exhibit more robust osteogenic but less adipogenic differentiation than BMMSCs $[6,37,39,41]$.

\section{The Superiority of UCMSCs in Clinical Application}

$\mathrm{BM}$ is considered as a traditional source of MSCs, and most of the knowledge concerning MSCs comes from BM studies. However, several limitations restrict the clinical application of BMMSCs. Harvesting BMMSCs involves an invasive and painful procedure, which can cause infection, bleeding, and chronic pain. Additionally, BMMSCs exhibit accelerated senescence significantly with age [38].

On the contrary, UCMSCs are obtained after delivery of a baby from a sample that would be discarded inevitably. The process is noninvasive, painless, and harmless for the mother and the baby. UC contains a significant amount of MSCs which can be easily collected and cultured $[34,40]$. In vitro, UCMSCs have greater expansion capability and faster growth rate $[3,17,39,40]$, indicating the advantage for rapid expansion and consequent downstream application.

UCMSCs express a lower level of HLA-class I than BMMSCs [40], suggesting the lower immunogenicity and the superiority for clinical use in HSCT. UCMSCs appear to 
have greater immunosuppressive effects [3], indicating their better role in the management of GVHD. Therefore, UC represents a good alternative source of MSCs and should not be discarded as medical waste.

\section{Clinical Application of MSCs in HSCT}

5.1. Promotion of Engraftment after HSCT. MSCs are crucial for the specialized BM microenvironment. Chemotherapy and radiotherapy prior to HSCT damage the BM stroma, and may result in delayed engraftment. In 2000, Koç et al. first reported that coinfusion of autologous BMMSCs at the time of HSCT can lead to rapid hematopoietic recovery [42]. Accordingly, 46 patients received allogeneic HSCs and MSCs from HLA-identical siblings, and most patients had prompt hematopoietic recovery without significant side effects [43]. The beneficial effects of MSCs on engraftment may relate to their supportive role in hematopoiesis.

Bacigalupo et al. demonstrated that BMMSCs from patients with severe aplastic anemia were deficient in the ability to inhibit T-cell proliferation and cytokine release, indicating the lack of MSC immunoprotection in the BM [44]. We found that BMMSCs derived from children with severe aplastic anemia exhibit poor potential of proliferation and differentiation [45]. Due to the possibility of BMMSC insufficiency, we cotransplanted UCMSCs along with HSCs in 2 children with severe aplastic anemia [4]. Both achieved faster hematopoietic engraftment without infusion-related toxicities.

5.2. Prevention and Treatment of Graft Failure. Apart from engraftment promotion, MSCs were reported to be also efficient in prevention and treatment of graft failure. In 2007, Le Blanc et al. reported cotransplantation of BMMSCs with HSCs to prevent rejection in 3 patients with previous graft failure or rejection [46]. All the patients achieved hematopoietic engraftment and $100 \%$ donor chimerism, suggesting the potential role of MSCs in the management of graft failure. As T cell depleted HSCT from a HLA-haploidentical relative is a feasible option for the patient who needs to undergo allogeneic HSCT but lacks a HLA-compatible donor, the report of Ball et al. provides promising results [47]. While there was a graft failure rate of $15 \%$ in 47 historical control patients, no graft failure occurred among the 14 children receiving coinfusion of BMMSCs during haploidentical HSCT.

5.3. Treatment of GVHD. GVHD involves increased secretion of inflammatory cytokines, activation of a variety of immune cells, and finally host tissue damage. Severe GVHD after allogeneic HSCT has a high mortality rate. MSCs can modulate immune responses and lead to resolving of GVHD. In 2004, Le Blanc et al. first reported that a girl with acute lymphoblastic leukemia received infusion of haploidentical BMMSCs to treat severe treatment-resistant grade IV acute GVHD after HSCT, and the clinical response was striking [48]. Thereafter, eight patients with steroid-refractory grades III-IV GVHD were treated with expanded MSCs from HLAidentical or mismatched donors, and the survival rate was significantly better than that of 16 comparable controls during the same period but not given MSCs [49]. In addition to the promising results of MSCs for GVHD, another important issue comes from their study: it is feasible and safe to use MSCs even from HLA-incompatible donors.

To date, clinical application of UCMSCs in the treatment of GVHD is very limited. According to our experience, we intravenously infused in vitro expanded UCMSCs four times into two recipients with severe steroid-resistant acute GVHD [3]. The clinical manifestations of GVHD dramatically improved after each UCMSC infusion, even without additional immunosuppressive therapies. Several studies demonstrated that MSCs of the donor can graft into the recipient's BM after allogeneic HSCT [43, 50, 51]. However, the marrow stroma remains host in origin after successful allogeneic HSCT [47, 52], suggesting that donor MSCs are eliminated in the recipient's BM after infusion. Therefore, more than one times of MSC infusion may be needed for patients with severe GVHD.

\section{Issues Related to Clinical Application of MSCs}

Because the number of MSCs obtained from the donor is not sufficient and MSCs have a great propensity for in vitro expansion, passaged cells are used extensively in experimental and clinical practice. Although karyotype analysis is not recommended for routine identification of MSCs, transforming events potentially leading to the establishment of a novel cell line may occur [5]. Additionally, MSCs may gradually lose their properties of early progenitor cells, including the abilities to proliferate and differentiate [53]. Thus, the conditions of MSC culture are very important for successful clinical use, and MSCs within six passages are suggested for clinical application [17].

MSCs may suppress host immunity against infectious agents, and it is uncertain whether viruses can be transmitted by MSCs from infected donors. Sundin et al. demonstrated that no viral DNA of cytomegalovirus, herpes simplex virus type 1 and type 2, Epstein-Barr virus and varicella zoster virus could be detected in MSCs isolated from healthy seropositive individuals [54]. Thus, the risk of herpes virus transmission is low when infusion of MSCs from healthy seropositive donors. However, cytopathological effects and intracellular viral antigens can be found after infection of cytomegalovirus and herpes simplex virus type 1 in vitro [54]. Therefore, MSCs may be susceptible in patients with viremia.

Coinfusion of in vitro expanded MSCs at the time of HSCT has been shown to be safe and feasible without immediate or late infusion-related toxicities [43, 47, 49], and patients given MSCs seem not to experience more infections [47]. However, the optimum MSC dose, the optimum number of infusions and the appropriate cell passages need to be further evaluated in large well-conducted clinical trials. In addition, UCMSCs are more primitive than BMMSCs. Although UCMSCs are easier to obtain without suffering to donors, the safety of these cells in human clinical application is still unclear due to the limited clinical experience. Besides, the quality of MSCs for human clinical use is crucial, but not 
all physicians have facilities to guarantee the quality of cells. "Off-the-shelf" MSCs from MSC banks may make MSCs more available for clinical application.

\section{Acknowledgments}

The study was supported by grants from Chung Shan Medical University Hospital (CSH-2012-A-004) and China Medical University Hospital (DMR-99-051).

\section{References}

[1] A. J. Friedenstein, I. I. Piatetzky-Shapiro, and K. V. Petrakova, "Osteogenesis in transplants of bone marrow cells," Journal of Embryology and Experimental Morphology, vol. 16, no. 3, pp. 381-390, 1966.

[2] B. H. Su, K. H. Wu, H. Y. Lin, M. H. Lin, C. T. Peng, and C. Tsai, "Umbilical cord-derived mesenchymal stem cells for severe bronchpulmonary dysplasia," Pediatrics, vol. 126, no. 125, pp. 1127-1133, 2011.

[3] K. H. Wu, C. K. Chan, C. Tsai et al., "Effective treatment of severe steroid-resistant acute graft-versus-host disease with umbilical cord-derived mesenchymal stem cells," Transplantation, vol. 91, no. 12, pp. 1412-1416, 2011.

[4] Y. H. Chao, C. Tsai, C. T. Peng et al., "Cotransplantation of umbilical cord MSCs to enhance engraftment of hematopoietic stem cells in patients with severe aplastic anemia," Bone Marrow Transplantation, vol. 46, no. 10, pp. 1391-1392, 2011.

[5] M. Dominici, K. Le Blanc, I. Mueller et al., "Minimal criteria for defining multipotent mesenchymal stromal cells. The International Society for Cellular Therapy position statement," Cytotherapy, vol. 8, no. 4, pp. 315-317, 2006.

[6] Z. Y. Zhang, S. H. Teoh, M. S. K. Chong et al., "Superior osteogenic capacity for bone tissue engineering of fetal compared with perinatal and adult mesenchymal stem cells," Stem Cells, vol. 27, no. 1, pp. 126-137, 2009.

[7] J. J. Minguell, A. Erices, and P. Conget, "Mesenchymal stem cells," Experimental Biology and Medicine, vol. 226, no. 6, pp. 507-520, 2001.

[8] G. Lazennec and C. Jorgensen, "Concise review: adult multipotent stromal cells and cancer: risk or benefit?" Stem Cells, vol. 26, no. 6, pp. 1387-1394, 2008.

[9] R. J. Deans and A. B. Moseley, "Mesenchymal stem cells: biology and potential clinical uses," Experimental Hematology, vol. 28, no. 8, pp. 875-884, 2000.

[10] Y. Ito, H. Hasauda, T. Kitajima, and T. Kiyono, "Ex vivo expansion of human cord blood hematopoietic progenitor cells using glutaraldehyde-fixed human bone marrow stromal cells," Journal of Bioscience \& Bioengineering, vol. 102, no. 5, pp. 467-469, 2006.

[11] C. L. da Silva, R. Gonçalves, K. B. Crapnell, J. M. S. Cabral, E. D. Zanjani, and G. Almeida-Porada, "A human stromalbased serum-free culture system supports the ex vivo expansion/maintenance of bone marrow and cord blood hematopoietic stem/progenitor cells," Experimental Hematology, vol. 33, no. 7, pp. 828-835, 2005.

[12] N. Li, P. Feugier, B. Serrurrier et al., "Human mesenchymal stem cells improve ex vivo expansion of adult human CD34 peripheral blood progenitor cells and decrease their allostimulatory capacity," Experimental Hematology, vol. 35, no. 3, pp. 507-515, 2007.

[13] G. Almeida-Porada, A. W. Flake, H. A. Glimp, and E. D. Zanjani, "Cotransplantation of stroma results in enhancement of engraftment and early expression of donor hematopoietic stem cells in utero," Experimental Hematology, vol. 27, no. 10, pp. 1569-1575, 1999.

[14] G. Almeida-Porada, C. D. Porada, N. Tran, and E. D. Zanjani, "Cotransplantation of human stromal cell progenitors into preimmune fetal sheep results in early appearance of human donor cells in circulation and boosts cell levels in bone marrow at later time points after transplantation," Blood, vol. 95, no. 11, pp. 3620-3627, 2000.

[15] W. A. Noort, A. B. Kruisselbrink, P. S. In't Anker et al., "Mesenchymal stem cells promote engraftment of human umbilical cord blood-derived $\mathrm{CD}^{+} 4^{+}$cells in NOD/SCID mice," Experimental Hematology, vol. 30, no. 8, pp. 870-878, 2002.

[16] R. Friedman, M. Betancur, L. Boissel, H. Tuncer, C. Cetrulo, and H. Klingemann, "Umbilical cord mesenchymal stem cells: adjuvants for human cell transplantation," Biology of Blood and Marrow Transplantation, vol. 13, no. 12, pp. 1477-1486, 2007.

[17] D. L. Troyer and M. L. Weiss, "Concise review: Wharton's Jellyderived cells are a primitive stromal cell population," Stem Cells, vol. 26, no. 3, pp. 591-599, 2008.

[18] I. Pelagiadis, H. Dimitriou, and M. Kalmanti, "Biologic characteristics of mesenchymal stromal cells and their clinical applications in pediatric patients," Journal of Pediatric Hematology/Oncology, vol. 30, no. 4, pp. 301-309, 2008.

[19] B. J. Jones and S. J. McTaggart, "Immunosuppression by mesenchymal stromal cells: from culture to clinic," Experimental Hematology, vol. 36, no. 6, pp. 733-741, 2008.

[20] S. Aggarwal and M. F. Pittenger, "Human mesenchymal stem cells modulate allogeneic immune cell responses," Blood, vol. 105, no. 4, pp. 1815-1822, 2005.

[21] A. Bartholomew, C. Sturgeon, M. Siatskas et al., "Mesenchymal stem cells suppress lymphocyte proliferation in vitro and prolong skin graft survival in vivo," Experimental Hematology, vol. 30, no. 1, pp. 42-48, 2002.

[22] C. Prevosto, M. Zancolli, P. Canevali, M. R. Zocchi, and A. Poggi, "Generation of $\mathrm{CD} 4^{+}$or $\mathrm{CD}^{+}$regulatory T cells upon mesenchymal stem cell-lymphocyte interaction," Haematologica, vol. 92, no. 7, pp. 881-888, 2007.

[23] A. Corcione, F. Benvenuto, E. Ferretti et al., "Human mesenchymal stem cells modulate B-cell functions," Blood, vol. 107, no. 1, pp. 367-372, 2006.

[24] R. Ramasamy, H. Fazekasova, E. W. F. Lam, I. Soeiro, G. Lombardi, and F. Dazzi, "Mesenchymal stem cells inhibit dendritic cell differentiation and function by preventing entry into the cell cycle," Transplantation, vol. 83, no. 1, pp. 71-76, 2007.

[25] I. Rasmusson, O. Ringdén, B. Sundberg, and K. Le Blanc, "Mesenchymal stem cells inhibit the formation of cytotoxic T lymphocytes, but not activated cytotoxic T lymphocytes or natural killer cells," Transplantation, vol. 76, no. 8, pp. 12081213, 2003.

[26] M. Di Nicola, C. Carlo-Stella, M. Magni et al., "Human bone marrow stromal cells suppress T-lymphocyte proliferation induced by cellular or nonspecific mitogenic stimuli," Blood, vol. 99, no. 10, pp. 3838-3843, 2002.

[27] S. Glennie, I. Soeiro, P. J. Dyson, E. W. F. Lam, and F. Dazzi, "Bone marrow mesenchymal stem cells induce division arrest anergy of activated T cells," Blood, vol. 105, no. 7, pp. 28212827, 2005.

[28] C. Campagnoli, I. A. Roberts, S. Kumar, P. R. Bennett, I. Bellantuono, and N. M. Fisk, "Identification of mesenchymal stem/progenitor cells in human first-trimester fetal blood, 
liver, and bone marrow," Blood, vol. 98, no. 8, pp. 2396-2402, 2001.

[29] P. V. Guillot, C. Gotherstrom, J. Chan, H. Kurata, and N. M. Fisk, "Human first-trimester fetal MSC express pluripotency markers and grow faster and have longer telomeres than adult MSC," Stem Cells, vol. 25, no. 3, pp. 646-654, 2007.

[30] P. S. In 't Anker, W. A. Noort, S. A. Scherjon et al., "Mesenchymal stem cells in human second-trimester bone marrow, liver, lung, and spleen exhibit a similar immunophenotype but a heterogeneous multilineage differentiation potential," Haematologica, vol. 88, no. 8, pp. 845-852, 2003.

[31] S. A. Wexler, C. Donaldson, P. Denning-Kendall, C. Rice, B. Bradley, and J. M. Hows, "Adult bone marrow is a rich source of human mesenchymal 'stem' cells but umbilical cord and mobilized adult blood are not," British Journal of Haematology, vol. 121, no. 2, pp. 368-374, 2003.

[32] S. Kern, H. Eichler, J. Stoeve, H. Klüter, and K. Bieback, "Comparative analysis of mesenchymal stem cells from bone marrow, umbilical cord blood, or adipose tissue," Stem Cells, vol. 24, no. 5, pp. 1294-1301, 2006.

[33] M. Yu, Z. Xiao, L. Shen, and L. Li, "Mid-trimester fetal blood-derived adherent cells share characteristics similar to mesenchymal stem cells but full-term umbilical cord blood does not," British Journal of Haematology, vol. 124, no. 5, pp. 666-675, 2004.

[34] M. Secco, E. Zucconi, N. M. Vieira et al., "Multipotent stem cells from umbilical cord: cord is richer than blood!," Stem Cells, vol. 26, no. 1, pp. 146-150, 2008.

[35] M. S. Tsai, S. M. Hwang, K. D. Chen et al., "Functional network analysis of the transcriptomes of mesenchymal stem cells derived from amniotic fluid, amniotic membrane, cord blood, and bone marrow," Stem Cells, vol. 25, no. 10, pp. 2511-2523, 2007.

[36] C. Götherström, A. West, J. Liden, M. Uzunel, R. Lahesmaa, and K. Le Blanc, "Difference in gene expression between human fetal liver and adult bone marrow mesenchymal stem cells," Haematologica, vol. 90, no. 8, pp. 1017-1026, 2005.

[37] W. Wagner, F. Wein, A. Seckinger et al., "Comparative characteristics of mesenchymal stem cells from human bone marrow, adipose tissue, and umbilical cord blood," Experimental Hematology, vol. 33, no. 11, pp. 1402-1416, 2005.

[38] K. Stenderup, J. Justesen, C. Clausen, and M. Kassem, "Aging is associated with decreased maximal life span and accelerated senescence of bone marrow stromal cells," Bone, vol. 33, no. 6, pp. 919-926, 2003.

[39] D. Baksh, R. Yao, and R. S. Tuan, "Comparison of proliferative and multilineage differentiation potential of human mesenchymal stem cells derived from umbilical cord and bone marrow," Stem Cells, vol. 25, no. 6, pp. 1384-1392, 2007.

[40] L. L. Lu, Y. J. Liu, S. G. Yang et al., "Isolation and characterization of human umbilical cord mesenchymal stem cells with hematopoiesis-supportive function and other potentials," Haematologica, vol. 91, no. 8, pp. 1017-1026, 2006.

[41] Y. J. Chang, D. T. Shih, C. P. Tseng, T. B. Hsieh, D. C. Lee, and S. M. Hwang, "Disparate mesenchyme-lineage tendencies in mesenchymal stem cells from human bone marrow and umbilical cord blood," Stem Cells, vol. 24, no. 3, pp. 679-685, 2006.

[42] O. N. Koç, S. L. Gerson, B. W. Cooper et al., "Rapid hematopoietic recovery after coinfusion of autologous-blood stem cells and culture-expanded marrow mesenchymal stem cells in advanced breast cancer patients receiving high-dose chemotherapy," Journal of Clinical Oncology, vol. 18, no. 2, pp. 307$316,2000$.
[43] H. M. Lazarus, O. N. Koc, S. M. Devine et al., "Cotransplantation of HLA-identical sibling culture-expanded mesenchymal stem cells and hematopoietic stem cells in hematologic malignancy patients," Biology of Blood and Marrow Transplantation, vol. 11, no. 5, pp. 389-398, 2005.

[44] A. Bacigalupo, M. Valle, M. Podestà et al., "T-cell suppression mediated by mesenchymal stem cells is deficient in patients with severe aplastic anemia," Experimental Hematology, vol. 33, no. 7, pp. 819-827, 2005.

[45] Y. H. Chao, C. T. Peng, H. J. Harn, C. K. Chan, and K. H. Wu, "Poor potential of proliferation and differentiation in bone marrow mesenchymal stem cells derived from children with severe aplastic anemia," Annals of Hematology, vol. 89, no. 7, pp. 715-723, 2010.

[46] K. Le Blanc, H. Samuelsson, B. Gustafsson et al., “Transplantation of mesenchymal stem cells to enhance engraftment of hematopoietic stem cells," Leukemia, vol. 21, no. 8, pp. 1733$1738,2007$.

[47] L. M. Ball, M. E. Bernardo, H. Roelofs et al., "Cotransplantation of ex vivo-expanded mesenchymal stem cells accelerates lymphocyte recovery and may reduce the risk of graft failure in haploidentical hematopoietic stem-cell transplantation," Blood, vol. 110, no. 7, pp. 2764-2767, 2007.

[48] K. Le Blanc, I. Rasmusson, B. Sundberg et al., "Treatment of severe acute graft-versus-host disease with third party haploidentical mesenchymal stem cells," The Lancet, vol. 363, no. 9419, pp. 1439-1441, 2004.

[49] O. Ringdén, M. Uzunel, I. Rasmusson et al., "Mesenchymal stem cells for treatment of therapy-resistant graft-versus-host disease," Transplantation, vol. 81, no. 10, pp. 1390-1397, 2006.

[50] E. M. Villaron, J. Almeida, N. López-Holgado et al., "Mesenchymal stem cells are present in peripheral blood and can engraft after allogeneic hematopoietic stem cell transplantation," Haematologica, vol. 89, no. 12, pp. 1421-1427, 2004.

[51] A. Poloni, P. Leoni, L. Buscemi et al., "Engraftment capacity of mesenchymal cells following hematopoietic stem cell transplantation in patients receiving reduced-intensity conditioning regimen," Leukemia, vol. 20, no. 2, pp. 329-335, 2006.

[52] N. Awaya, K. Rupert, E. Bryant, and B. Torok-Storb, "Failure of adult marrow-derived stem cells to generate marrow stroma after successful hematopoietic stem cell transplantation," Experimental Hematology, vol. 30, no. 8, pp. 937-942, 2002.

[53] A. Banfi, A. Muraglia, B. Dozin, M. Mastrogiacomo, R. Cancedda, and R. Quarto, "Proliferation kinetics and differentiation potential of ex vivo expanded human bone marrow stromal cells: implications for their use in cell therapy," Experimental Hematology, vol. 28, no. 6, pp. 707-715, 2000.

[54] M. Sundin, C. Örvell, I. Rasmusson, B. Sundberg, O. Ringdén, and K. Le Blanc, "Mesenchymal stem cells are susceptible to human herpesviruses, but viral DNA cannot be detected in the healthy seropositive individual," Bone Marrow Transplantation, vol. 37, no. 11, pp. 1051-1059, 2006. 


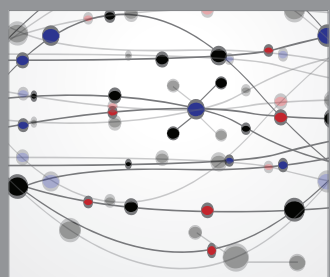

The Scientific World Journal
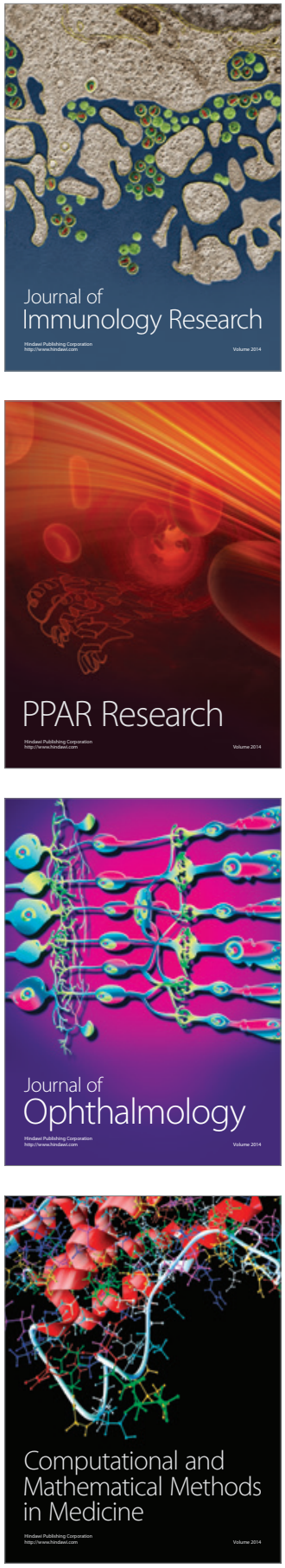

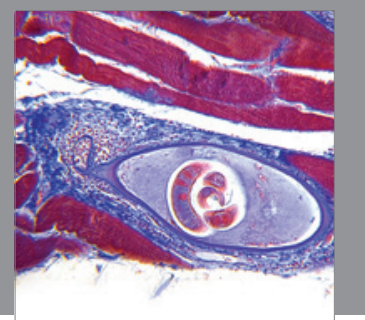

Gastroenterology

Research and Practice
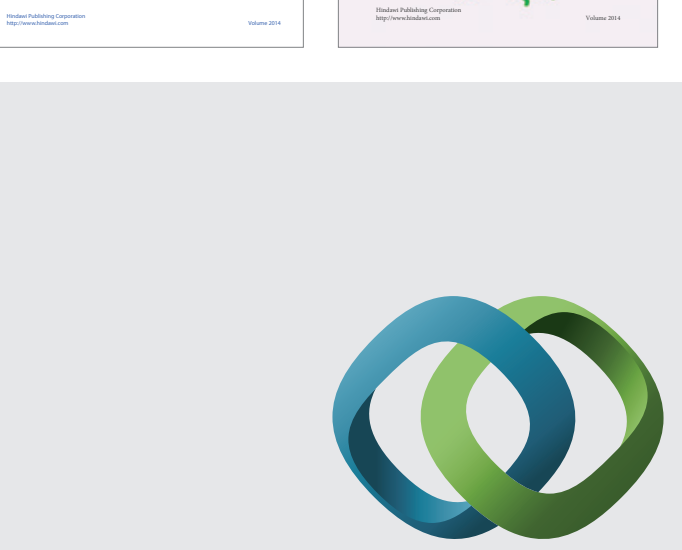

\section{Hindawi}

Submit your manuscripts at

http://www.hindawi.com


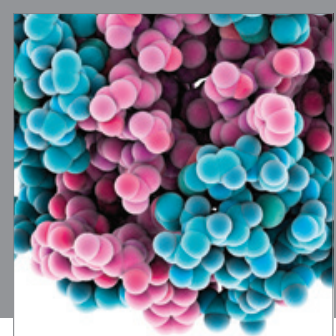

Journal of
Diabetes Research

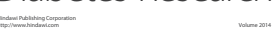

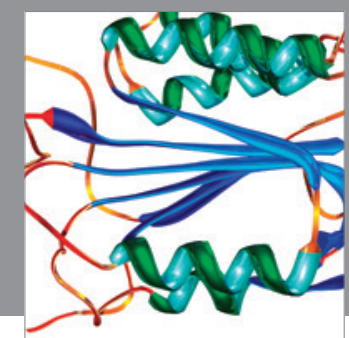

Disease Markers
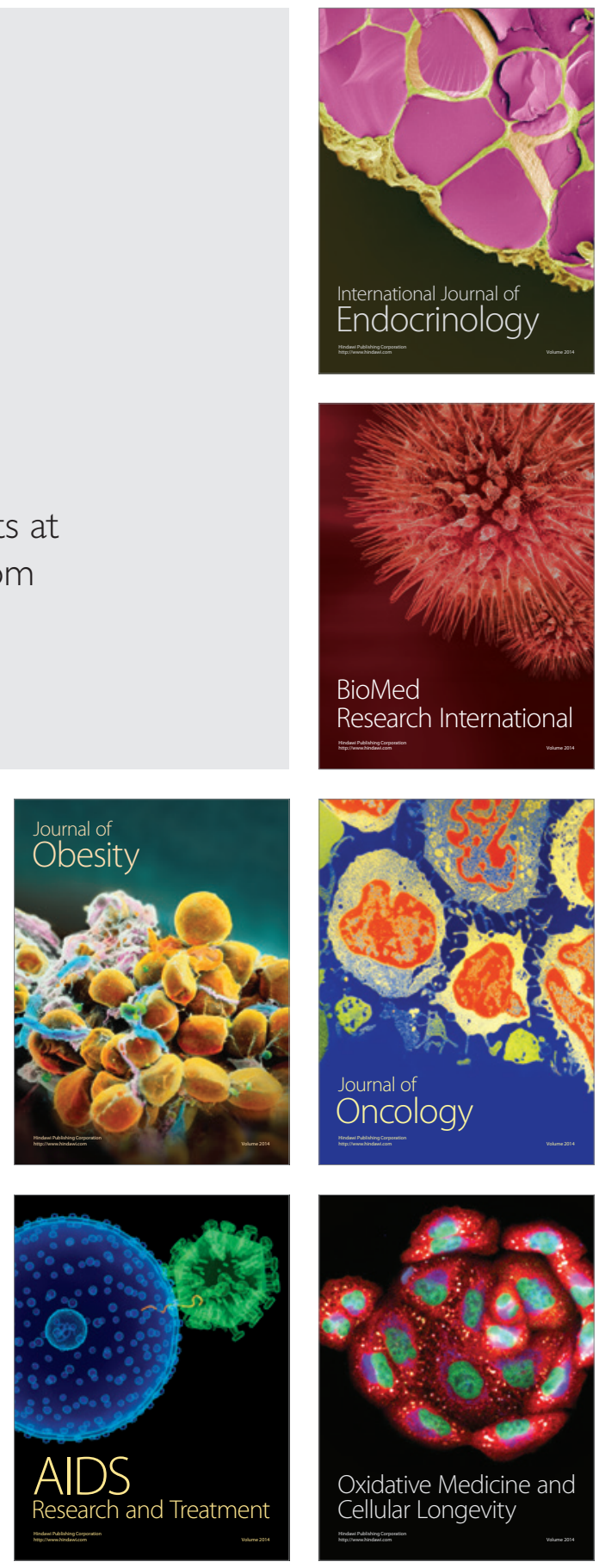